\title{
Energy-Chirp Compensation in a Laser Wakefield Accelerator
}

\author{
A. Döpp, ${ }^{1,2}$ C. Thaury, ${ }^{1}$ E. Guillaume, ${ }^{1}$ F. Massimo, ${ }^{1}$ A. Lifschitz, ${ }^{1}$ I. Andriyash, ${ }^{1,3}$ J.-P. Goddet, ${ }^{1}$ \\ A. Tazfi, ${ }^{1}$ K. Ta Phuoc, ${ }^{1}$ and V. Malka ${ }^{1,3}$ \\ ${ }^{1}$ LOA, ENSTA ParisTech-CNRS-École Polytechnique-Université Paris-Saclay, \\ 828 Boulevard des Maréchaux, 91762 Palaiseau Cedex, France \\ ${ }^{2}$ Ludwig-Maximilians-Universität München, Am Coulombwall 1, 85748 Garching, Germany \\ ${ }^{3}$ Department of Physics and Complex Systems, Weizmann Institute of Science, Rehovot 76100, Israel
}

(Received 31 January 2017; published 17 August 2018)

\begin{abstract}
The energy spread in laser wakefield accelerators is primarily limited by the energy chirp introduced during the injection and acceleration processes. Here, we propose the use of longitudinal density tailoring to reduce the beam chirp at the end of the accelerator. Experimental data sustained by quasi-3D particle-incell simulations show that broadband electron beams can be converted to quasimonoenergetic beams of $\leq 10 \%$ energy spread while maintaining a high charge of more than $120 \mathrm{pC}$. In the linear and quasilinear regimes of wakefield acceleration, the method could provide even lower, subpercent level, energy spread.
\end{abstract}

DOI: 10.1103/PhysRevLett.121.074802

Laser wakefield acceleration is an aspiring technology to produce femtosecond bunches of highly relativistic electrons in a compact way [1-3]. While the high field gradients permit acceleration of electrons to hundreds of megaelectronvolt on a millimeter scale [4], they also cause a large energy spread between electrons that are trapped and accelerated at different times. Nonetheless, if the trapping conditions are only met during a short moment, all electrons experience similar accelerating fields and the electron beams become quasimonoenergetic [5-7]. To enter this regime, the accelerator needs to be operated at a relatively low plasma density so that injection is caused by self-focusing and self-compression of the laser pulse [8]. Once a certain charge has been trapped inside the wake, beam-loading prohibits further injection [9].

Unfortunately, it is difficult to achieve good stability and tunability if injection relies on the nonlinear laser propagation [10]. This is why a number of controlled injection schemes have been developed to provide favorable trapping conditions at a defined position and time [11]. The most prominent techniques are arguably colliding-pulse injection [12] and shock-front injection [13], which allow for accurate tuning of the electron beam energy while maintaining a low energy spread. But electron beams from such localized injection schemes typically contain an order of magnitude less charge than broadband beams from selfinjection, ionization-induced injection [14,15], or density downramp injection $[16,17]$.

However, the beam energy spread is not solely determined by the injection process. In a laser wakefield accelerator, a plasma wave is formed behind the laser pulse which propagates at the group velocity $v_{g}$. In contrast, highly relativistic electrons with a Lorentz factor $\gamma \gg 1$ propagate at nearly the speed of light in vacuum $\left(v_{e} \simeq c_{0}\right)$ and will gain on the laser beam and its wake during the acceleration process. When new electrons get subsequently injected at the back of the wake, this results in a clear relation between position and energy. Initially, this relation can often be described with a linear chirp $\alpha$ and the beam energy spread is essentially $\Delta E \sim \sigma_{z} \times|\alpha|$, where $\sigma_{z}$ is the bunch length.

Another result of the mismatch between laser and electron velocity is that electrons gradually propagate from the accelerating phase of the wake into the decelerating region. This dephasing is mostly known as a major limitation for the energy gain in laser wakefield accelerators. But as a side effect, dephasing also reduces the beam energy spread: During the dephasing process, electrons at the bunch head start to decelerate, while trailing electrons still gain energy. If the accelerator length is tuned closely to the dephasing length, this effect naturally compensates the linear energy chirp. In experiment, such alignment can be achieved by changing the background plasma density, but this will also affect the laser propagation, plasma wake formation and electron injection. Gas cells avoid this drawback as their length can be adjusted at constant plasma density in order to match the dephasing length $[18,19]$. Still, this will only compensate the chirp for a fixed beam energy and accelerator length. If the acceleration is stopped before, e.g., if the dephasing length is longer than the pump depletion length or the effective Rayleigh length, the electron beam spread remains increased due to the nonzero chirp.

In recent theoretical work, we have discussed different ways how longitudinal plasma density tailoring can be used to manipulate electron beam properties $[20,21]$. These techniques were then applied in experiments to produce electron beams with reduced beam divergence [22] and 
increased beam energy [23]. In this Letter, we discuss how a similar approach can be used to reduce the beam energy chirp, and with it the beam energy spread, at the end of the wakefield accelerator.

The basic principle of the scheme is illustrated in Fig. 1. Most injection schemes such as self-injection, downramp injection, or ionization-induced injection produce electron beams with negative chirp. We propose to use a transition to higher plasma density to increase the acceleration of the rear part of the bunch, while the front is less accelerated and later decelerated. As a result, the beam rotates in the $\left(z, p_{z}\right)$ phase space, shown in the lower part of Fig. 1. Given that the beam energy is mainly determined by the longitudinal momentum $p_{z}$, this reduces both the energy chirp and the energy spread [24]. Please note that a similar scenario was studied in simulations by $\mathrm{Hu}$ et al. [25].

We have demonstrated the method in experiments using the 40 TW SALLE JAUNE Ti: Sa laser system at Laboratoire d'Optique Appliquée, which delivers pulses of $1.2 \mathrm{~J}$ energy on target with $30 \mathrm{fs}$ duration and $800 \mathrm{~nm}$ central wavelength. The laser is focused at the entrance of a supersonic helium gas jet using a $f / 10$ off-axis parabola. The focal spot size is $15 \mu \mathrm{m}$, with a peak intensity on target of $I=1.0 \times 10^{19} \mathrm{~W} / \mathrm{cm}^{2}$, which corresponds to a normalized vector potential $a_{0}=2.2$. The plasma density distribution is measured using a Nomarski-type interferometer [26]. Electron beams are characterized with a magnet spectrometer, giving information about electron beam charge, divergence, and their energy spectrum from $70 \mathrm{MeV}$ onwards.

The density profile consists of a linearly rising and then falling slope of about $1 \mathrm{~mm}$ length each, peaking at $1.6 \times 10^{19} \mathrm{~cm}^{-3}$, see Fig. 2(a) [27]. While the laser

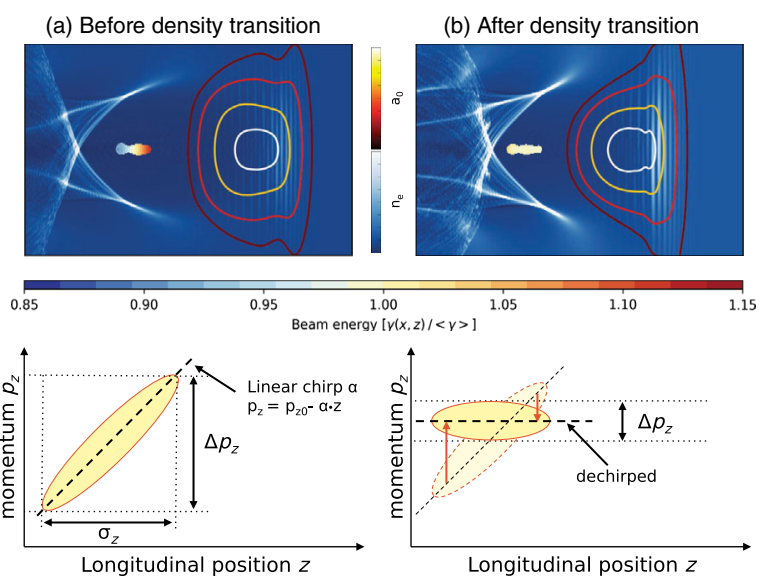

FIG. 1. Illustration of chirp reduction as a result of a density transition. Upper part: Plasma density (blue color map), laser intensity (isolevels), and beam energy before (a) and after (b) a density transition as calculated with PIC simulations. Lower part: Sketch of the $\left(z, p_{z}\right)$ phase space for both cases. The beam is initially chirped (dashed line) and therefore electrons of different energy are located at different phases of the wakefield. Using the density transition, the phase space ellipse (yellow) can be rotated, thus reducing both chirp and beam energy spread. propagates through the first part of the jet, the increasing plasma density causes the phase velocity of the wake to augment as well [28]. This will prevent injection at this stage of the interaction. In contrast, from the middle of the jet on, electrons are expected to be injected via density gradient injection [17]. In accordance with this, the measured electron beams are spectrally broadband [see upper part of Fig. 2(b)]. While the density downramp allows electrons to get trapped easily, it also reduces the effective acceleration length and field. Accordingly, the measured cutoff energy of $122 \pm 9 \mathrm{MeV}$ is lower than what would be expected for a flat density profile at the same peak density ( $200 \mathrm{MeV}$ using the scalings from Lu et al. [29]). The beam charge is $146 \pm 22 \mathrm{pC}$, with a divergence of $8 \pm 3 \mathrm{mrad}$. Because of the broadband nature of the electron beam, the spectral charge density typically remains below $3 \mathrm{pC} / \mathrm{MeV}$.

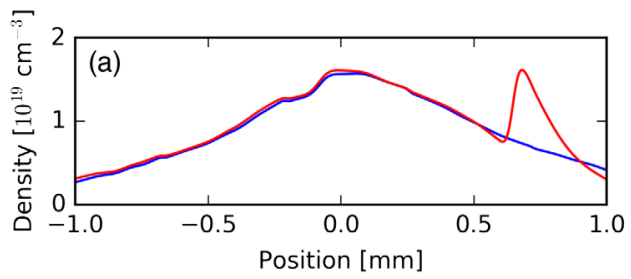

(b)
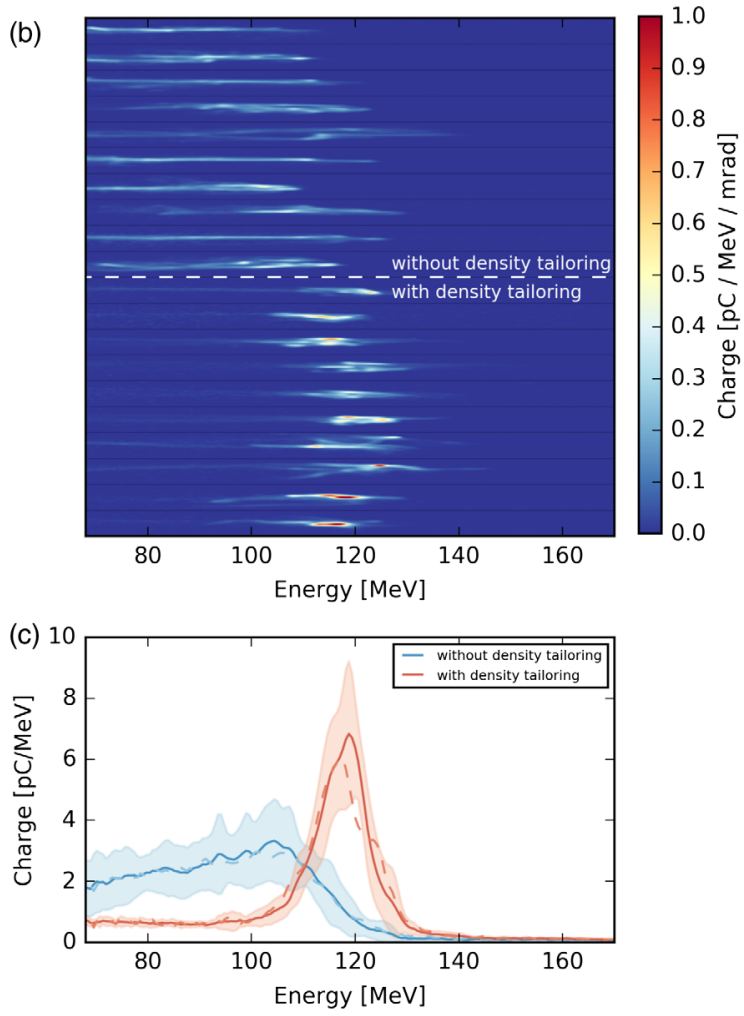

FIG. 2. Experimental data. (a) Density profile measurements. (b) Angularly resolved single-shot electron spectra for an unperturbed jet (above) and with density tailoring using a shock front (below). (c) Integrated electron spectra for both cases (dashed lines for average spectra, solid lines for average spectra with corrected peak energies). 

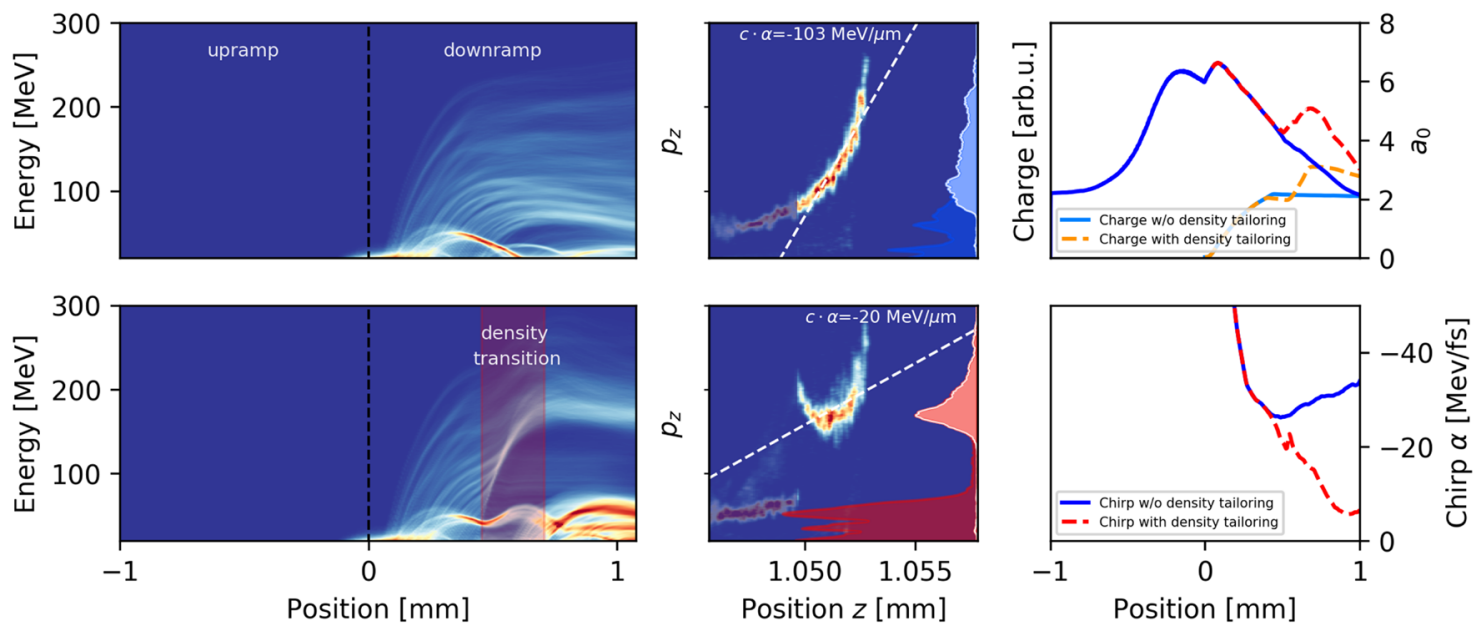

FIG. 3. Results from 3D PIC simulations. Left: Evolution of the electron beam spectrum in the laser wakefield accelerator without density tailoring (top) and with density tailoring (bottom). The corresponding $\left(z, p_{z}\right)$ phase spaces at the end of the accelerator are shown in the panel to the right. Light colors show the integrated spectrum within the rephasing region, shaded colors show the spectrum of the entire beam. Injection and laser dynamics are plotted in the top right, the evolution of the beam energy chirp is shown on the bottom right.

To create a density transition, a silicon wafer is used, which is mounted on a motorized stage at the rear part of the jet. The obstacle in the supersonic gas flow leads to the formation of a shock front that travels downstream [13]. Placed at the leaving side of the jet, this results in a sharp upward density transition along the laser axis of propagation. The longitudinal position of this transition can be adjusted by moving the blade. While the density at the shock is similar to the density at the center of the jet, the plasma density rapidly decreases behind the shock, hence terminating the acceleration process.

The beam energy distribution changes drastically once the density transition is introduced [see Figs. 2(b) and 2(c)]. With the transition located at $0.7 \mathrm{~mm}$ behind the center of the jet, the broad energy spectrum is converted into a distribution that peaks at $117 \pm 12 \mathrm{MeV}$, with an energy spread of less than 10 percent. The beam charge is similar, but slightly lower than in to the nonperturbed case $(123 \pm 18 \mathrm{pC})$ and the spectral charge density at the peak increases to over $6 \mathrm{pC} / \mathrm{MeV}$. The beam divergence remains unaffected $(8 \pm 3 \mathrm{mrad})$. As expected, the final beam spectrum is sensitive to the position of the density transition. When the transition occurs too early, it disturbs the electron injection process and the electron beam is essentially lost. The further the silicon wafer is moved outside of the jet, the less pronounced the narrowing of the spectrum becomes, until the electron distribution resembles the case without density tailoring [30].

To gain more insight in the physics that lead to this result, the experiment is modeled using the quasi-3D particle-in-cell code CALDER-CIRC [31]. According to the experiment, a Gaussian laser pulse is initialized at $z=$ $-1 \mathrm{~mm}$ with $w_{0}=15 \mu \mathrm{m}, \tau=30 \mathrm{fs}$, and $a_{0}=2.2$, while the plasma density profile is defined based on the experimentally measured profiles, with a peak density of $1.6 \times 10^{19} \mathrm{~cm}^{-3}$ at $z=0 \mathrm{~mm}$. The resolution is $\Delta z=0.25 k_{0}^{-1}, \Delta r=1.0 k_{0}^{-1}$, and $c \Delta t=0.94 \Delta z$, with two Fourier modes $(m=0-1)$ in the poloidal direction and 50 particles per cell. The results are summarized in Fig. 3.

First, no electrons are accelerated during propagation along the density upramp. At the same time, the laser is self-focusing and self-compressing, reaching a peak vector potential $a_{0}=6.6$ in vicinity of the density peak at $z=0 \mathrm{~mm}$. Once the laser enters the downramp, the wakefield starts to expand and electrons are trapped and accelerated inside the bubble. The continuous injection leads to a large energy spread. But as observed in the experiment, the electron beam spectrum changes significantly using density tailoring. In this case, the spectrum exhibits a clear peak at $168 \mathrm{MeV}$ and the energy spread within the same section of the beam reduces from of $139 \mathrm{MeV}$ at full width at half maximum to $39 \mathrm{MeV}$.

The simulations show that this behavior is primarily caused by a reduction in the energy chirp of the beam. As shown in Fig. 3, the electron beam from downramp injection has a linear chirp of more than $30 \mathrm{MeV} / \mathrm{fs}$ at the end of the simulation. We find that the density transition reduces the linear chirp significantly, to less than $10 \mathrm{MeV} / \mathrm{fs}$. As conceived, this is the result of enforced acceleration fields at the back of the laser wakefield. But the field structure is not ideal. Even though this leads to nearly chirp-free regions in the center of the beam, the bunch head and tail have a higher energy, thus leading to nonlinear chirp components.

Another observation of the experiments is that the bunch charge above the detection threshold of $70 \mathrm{MeV}$ is comparable for both cases. Indeed, when considering the 
charge above this threshold, the density-tailored case yields 88 percent of the unperturbed charge, which is very similar to the experiment (84\%). This is significantly higher than in first demonstrations of electron rephasing with self-injected beams [23]. While a part of the low-energy tail is still lost during the density upramp, the charge below $70 \mathrm{MeV}$ is even higher with density tailoring, which is due to injection in the steep density downramp at the rear side of the shock front.

The simulations therefore support the conclusion that the energy spread reduction observed in the experiment is due to a significant reduction in energy chirp. Thus, the scheme is a promising new approach to generate quasimonoenergetic electron beams of high charge with laser wakefield accelerators. Note that it is preferable to compensate the beam chirp once the entire beam has advanced inside the ion cavity (as in Fig. 1), to prevent loosing electrons that are injected shortly before the density transition.

In the future, it would be even more attractive if density tailoring could provide complete phase space control, for instance, to reach subpercent level energy spread. However, it is difficult to reach such performance in the nonlinear blowout regime, both in simulations and experiments. This is because fine-tuning of the wakefields is difficult due to complex interplay of plasma density and laser evolution. Furthermore, electron blowout leads to sawtoothlike, negative-gradient wakefields [29], which cannot compensate for positive chirp. This occurs, e.g., in shock-front injection and limits the minimal energy spread of this otherwise high-quality injection technique. As we briefly discuss in the following, operating the wakefield accelerator in the (quasi)linear regime may help to address these issues.

Using a setup similar to a laser-plasma lens [20], the remaining part of the accelerating laser pulse or a second laser pulse could be used to create a (quasi)linear wakefield in a subsequent, second gas target that solely serves the purpose of dechirping the beam. In the case of a single pulse, the intensity of the latter can be adjusted by changing the distance between the accelerator and dechirping stage, while the effective accelerating fields can be fine-tuned with the density profile. As an illustration of this scheme's potential, Fig. 4 shows results based on fluid model calculations [32]. A Gaussian gas density profile of variable width and peak density is assumed for the dechirping stage, which is typical for targets based on sonic gas jets. Starting from a beam with a linear chirp of $\alpha_{\text {initial }}=-0.6 \mathrm{MeV} / \mathrm{fs}$, the chirp can be almost entirely compensated $\left(\alpha_{\text {final }}=-0.03 \mathrm{MeV} / \mathrm{fs}\right)$ and the rms energy spread is lowered to $0.4 \%$ (1.1\% FWHM). Additionally, the higher order chirp and with it the longitudinal emittance are reduced. The results indicate that such a chirp compensation in a longitudinally tailored plasma could be an alternative to other proposals, like chirp mitigation in density modulated plasmas [33], in order to reach subpercent level energy spread beams in laser wakefield accelerators.
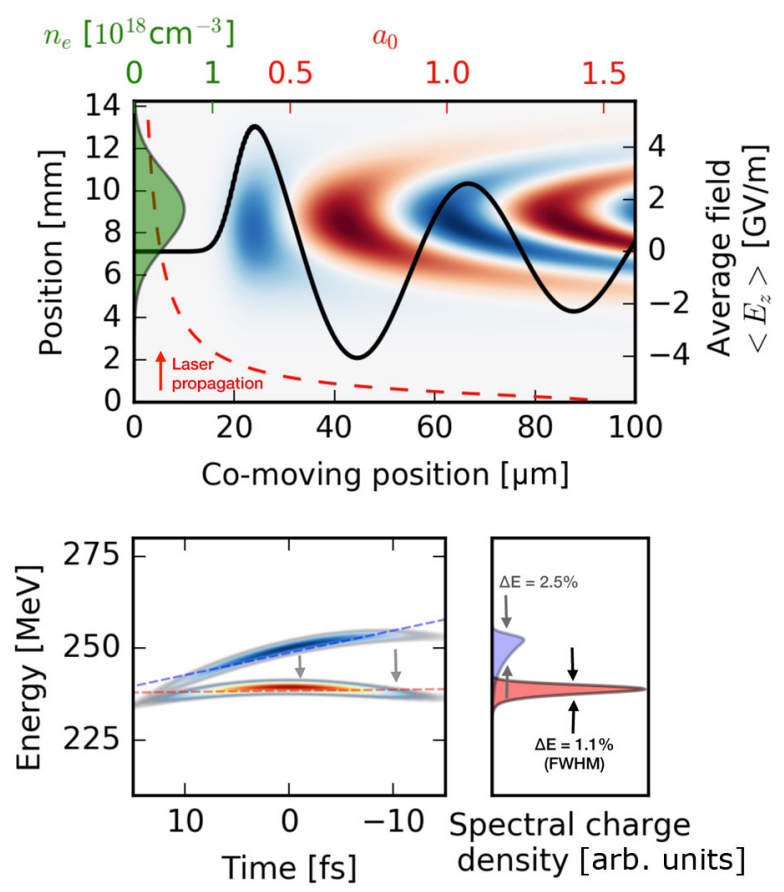

FIG. 4. Simulated chirp compensation in the linear wakefield regime. Upper plot: Wakefield (color map) and average electric field (black line) in the wakefield created by a laser pulse with $a_{0}(z)$ (dashed red line) in a gas jet with Gaussian density distribution (shaded green area). Bottom: Initial electron beam phase space and spectrum (blue) and chirp-compensated beam (red).

In conclusion, we have presented results on energy chirp compensation in density-tailored laser wakefield accelerators. The results extend the laser-plasma lensing and rephasing concepts to the production of low energy spread electron beams. We experimentally demonstrated an energy spread reduction of a broadband electron beam to less than 10 percent, while maintaining a high charge of about $120 \mathrm{pC}$ and a divergence of $8 \mathrm{mrad}$. The method facilitates the production of highly charged bunches of monoenergetic electrons and is simple to implement in existing setups using either gas jets or double compartment gas cells [34]. This kind of beam is of immediate interest for laser-driven x-ray sources, such as inverse Compton sources [35,36], and free-electron lasing experiments [37,38]. The latter would especially benefit from the increased spectral charge density. Furthermore, density tailoring in the quasilinear regime may lead to the production of even lower, subpercent energy spread beams.

This project has received funding from the European Union's Horizon 2020 Research and Innovation programme under Grant No. 730871 (project ARIES) and from the European Research Council (ERC) under Grant No. 339128 (project X-Five). We acknowledge also the support from the Agence Nationale pour la Recherche through the projects FEMTOMAT (ANR-13-BS04-0002) and LUCELX project (ANR-13-BS04-0011). The authors 
gratefully acknowledge the Gauss Centre for Supercomputing e.V. for funding this project by providing computing time on the GCS Supercomputer SuperMUC at Leibniz Supercomputing Centre under Project No. pn69ri. A. D. acknowledges support by DFG through the Cluster of Excellence Munich-Centre for Advanced Photonics (MAP EXC 158) and thanks the OSIRIS consortium (IST/UCLA) for access to the OSIRIS code. F. M. received support by the European Union's Horizon 2020 research and innovation programme under Grant EuPRAXIA No. 653782.

[1] V. Malka, J. Faure, Y. A. Gauduel, E. Lefebvre, A. Rousse, and K. T. Phuoc, Nat. Phys. 4, 447 (2008).

[2] E. Esarey, C. B. Schroeder, and W. P. Leemans, Rev. Mod. Phys. 81, 1229 (2009).

[3] S. M. Hooker et al., Nat. Photonics 7, 775 (2013).

[4] V. Malka, S. Fritzler, E. Lefebvre, M.-M. Aleonard, F. Burgy, J. P. Chambaret, J.-F. Chemin, K. Krushelnick, G. Malka, and S. Mangles, Science 298, 1596 (2002).

[5] S. P. D. Mangles, C. D. Murphy, Z. Najmudin, A. G. R. Thomas, J. L. Collier, A. E. Dangor, E. J. Divall, P. S. Foster, J. G. Gallacher, C. J. Hooker et al., Nature (London) 431, 535 (2004).

[6] C. G. R. Geddes, C. Toth, J. van Tilborg, E. Esarey, C. B. Schroeder, D. Bruhwiler, C. Nieter, J. Cary, and W. P. Leemans, Nature (London) 431, 538 (2004).

[7] J. Faure, Y. Glinec, A. Pukhov, S. Kiselev, S. Gordienko, E. Lefebvre, J. P. Rousseau, F. Burgy, and V. Malka, Nature (London) 431, 541 (2004).

[8] S. Kalmykov, S. A. Yi, V. Khudik, and G. Shvets, Phys. Rev. Lett. 103, 135004 (2009).

[9] C. Benedetti, C. B. Schroeder, E. Esarey, F. Rossi, and W. P. Leemans, Phys. Plasmas 20, 103108 (2013).

[10] A. Döpp, B. Mahieu, A. Lifschitz, C. Thaury, A. Doche, E. Guillaume, G. Grittani, O. Lundh, M. Hansson, J. Gautier et al., Light Sci. Appl. 6, e17086 (2017).

[11] V. Malka, Phys. Plasmas 19, 055501 (2012).

[12] J. Faure, C. Rechatin, A. Norlin, A. Lifschitz, Y. Glinec, and V. Malka, Nature (London) 444, 737 (2006).

[13] K. Schmid, A. Buck, C. M. S. Sears, J. M. Mikhailova, R. Tautz, D. Herrmann, M. Geissler, F. Krausz, and L. Veisz, Phys. Rev. ST Accel. Beams 13, 091301 (2010).

[14] C. E. Clayton, J. E. Ralph, F. Albert, R. A. Fonseca, S. H. Glenzer, C. Joshi, W. Lu, K. A. Marsh, S. F. Martins, W. B. Mori et al., Phys. Rev. Lett. 105, 105003 (2010).

[15] E. Guillaume, A. Döpp, C. Thaury, A. Lifschitz, J. P. Goddet, A. Tafzi, F. Sylla, G. Iaquanello, T. Lefrou, P. Rousseau et al., Phys. Rev. ST Accel. Beams 18, 061301 (2015).

[16] S. Bulanov, N. Naumova, F. Pegoraro, and J. Sakai, Phys. Rev. E 58, R5257 (1998).

[17] C. G. R. Geddes, K. Nakamura, G. R. Plateau, C. Toth, E. Cormier-Michel, E. Esarey, C. B. Schroeder, J. R. Cary, and W. P. Leemans, Phys. Rev. Lett. 100, 215004 (2008).
[18] S. Corde, A. Lifschitz, G. Lambert, K. T. Phuoc, X. Davoine, R. Lehe, D. Douillet, A. Rousse, V. Malka, and C. Thaury, Nat. Commun. 4, 1501 (2013).

[19] M. Heigoldt, A. Popp, K. Khrennikov, J. Wenz, S. W. Chou, S. Karsch, S. I. Bajlekov, S. M. Hooker, and B. Schmidt, Phys. Rev. ST Accel. Beams 18, 121302 (2015).

[20] R. Lehe, C. Thaury, E. Guillaume, A. Lifschitz, and V. Malka, Phys. Rev. ST Accel. Beams 17, 121301 (2014).

[21] A. Döpp, E. Guillaume, C. Thaury, A. Lifschitz, K. Ta Phuoc, and V. Malka, Phys. Plasmas 23, 056702 (2016).

[22] C. Thaury, E. Guillaume, A. Döpp, R. Lehe, A. Lifschitz, K. T. Phuoc, J. Gautier, J. P. Goddet, A. Tafzi, A. Flacco et al., Nat. Commun. 6, 6860 (2015).

[23] E. Guillaume, A. Döpp, C. Thaury, K. Ta Phuoc, A. Lifschitz, G. Grittani, J. P. Goddet, A. Tafzi, S. W. Chou, L. Veisz et al., Phys. Rev. Lett. 115, 155002 (2015).

[24] See Supplemental Material at http://link.aps.org/ supplemental/10.1103/PhysRevLett.121.074802 for information on these simulations.

[25] R. Hu, H. Lu, Y. Shou, C. Lin, H. Zhuo, C.-e. Chen, and X. Yan, Phys. Rev. Accel. Beams 19, 091301 (2016).

[26] R. Benattar, C. Popovics, and R. Sigel, Rev. Sci. Instrum. 50, 1583 (1979).

[27] See Supplemental Material at http://link.aps.org/ supplemental/10.1103/PhysRevLett.121.074802 for more information on the interferometry measurements.

[28] J. Faure, C. Rechatin, O. Lundh, L. Ammoura, and V. Malka, Phys. Plasmas 17, 083107 (2010).

[29] W. Lu, M. Tzoufras, C. Joshi, F. Tsung, W. Mori, J. Vieira, R. Fonseca, and L. Silva, Phys. Rev. ST Accel. Beams 10, 061301 (2007).

[30] See Supplemental Material at http://link.aps.org/ supplemental/10.1103/PhysRevLett.121.074802 for additional plots.

[31] A. Lifschitz, X. Davoine, E. Lefebvre, J. Faure, C. Rechatin, and V. Malka, J. Comput. Phys. 228, 1803 (2009).

[32] See Supplemental Material at http://link.aps.org/ supplemental/10.1103/PhysRevLett.121.074802 for details.

[33] R. Brinkmann, N. Delbos, I. Dornmair, M. Kirchen, R. Assmann, C. Behrens, K. Floettmann, J. Grebenyuk, M. Gross, S. Jalas et al., Phys. Rev. Lett. 118, 214801 (2017).

[34] O. Kononenko, N. C. Lopes, J. M. Cole, C. Kamperidis, S. P. D. Mangles, Z. Najmudin, J. Osterhoff, K. Poder, D. Rusby, D. R. Symes et al., Nucl. Instrum. Methods Phys. Res., Sect. A 829, 125 (2016).

[35] K. Khrennikov, J. Wenz, A. Buck, J. Xu, M. Heigoldt, L. Veisz, and S. Karsch, Phys. Rev. Lett. 114, 195003 (2015).

[36] A. Döpp, E. Guillaume, C. Thaury, J. Gautier, I. Andriyash, A. Lifschitz, V. Malka, A. Rousse, and K. T. Phuoc, Plasma Phys. Controlled Fusion 58, 034005 (2016).

[37] A. R. Maier, A. Meseck, S. Reiche, C. B. Schroeder, T. Seggebrock, and F. Gruner, Phys. Rev. X 2, 031019 (2012).

[38] M. E. Couprie, C. Benabderrahmane, P. Betinelli, F. Bouvet, A. Buteau, L. Cassinari, J. Daillant, J. C. Denard, P. Eymard, B. Gagey et al., J. Phys. Conf. Ser. 425, 072001 (2013). 